\title{
Selection of an Interval for Variable Shape Parameter in Approximation by Radial Basis Functions
}

\author{
Jafar Biazar and Mohammad Hosami \\ Department of Applied Mathematics, Faculty of Mathematical Sciences, University of Guilan, P.O. Box 41335-1914, \\ Rasht 41938 22697, Iran \\ Correspondence should be addressed to Jafar Biazar; jafar.biazar@gmail.com
}

Received 29 April 2016; Revised 12 July 2016; Accepted 20 July 2016

Academic Editor: Zhangxin Chen

Copyright ( $\odot 2016$ J. Biazar and M. Hosami. This is an open access article distributed under the Creative Commons Attribution License, which permits unrestricted use, distribution, and reproduction in any medium, provided the original work is properly cited.

\begin{abstract}
In radial basis function approximation, the shape parameter can be variable. The values of the variable shape parameter strategies are selected from an interval which is usually determined by trial and error. As yet there is not any algorithm for determining an appropriate interval, although there are some recipes for optimal values. In this paper, a novel algorithm for determining an interval is proposed. Different variable shape parameter strategies are examined. The results show that the determined interval significantly improved the accuracy and is suitable enough to count on in variable shape parameter strategies.
\end{abstract}

\section{Introduction}

Radial basis function methods are the tools for interpolating a multivariate data set, approximating a function, and solving partial differential equations [1-5]. A radial basis function, say $\varphi(r)$, is a continuous univariate function that has been realized by composition with the Euclidean norm in $\mathbb{R}^{d}$. Radial basis functions (RBFs) may contain a parameter called the shape parameter. Most well-known RBFs have a shape parameter, such as Multiquadric (MQ), Inverse Multiquadric (IMQ), and Gaussian parameters. A well-known RBF without a shape parameter is thin-plate spline (TPS). This class of RBFs approximates with polynomial convergence rate, but three mentioned RBFs approximate with spectral convergence rate. This point leads to applying RBFs with a shape parameter, even though we know that the value of the shape parameter is an issue. In these radial basis functions, the value of the shape parameter plays an important role for the accuracy of the procedure. An important problem is determining the optimal value for the shape parameter. Several articles have been dedicated to introducing different algorithms to compute a constant value, as an optimal or a good value, for the shape parameter [6-15]. Kansa and Carlson showed that instead of a fixed shape parameter variable shape parameters are also useful [16]. They distribute some values in an interval to use them in variable shape parameter. Moreover, there are some articles applying the variable shape parameter in various problems [17-20]. But in many of them there is not any criterion to select the interval of the shape parameters. In fact the selected interval to distribute the shape parameters is user-defined as the selection of the value in constant shape parameter.

In this paper, we focus on the selection of a valid interval in variable shape parameter. An algorithm is suggested to obtain this interval. To show the efficiency of the proposed interval, it is investigated in different variable shape parameter strategies.

\section{Shape Parameter in MQ-RBF Approximation}

In the RBF methods, a function $u(x)$ is approximated by $u^{*}(x)$ as in the following:

$$
u^{*}(x)=\sum_{j=1}^{N} \lambda_{j} \varphi\left(\left\|x-x_{j}\right\|_{2}\right) .
$$

By interpolation conditions

$$
u\left(x_{i}\right)=f_{i}, \quad j=1, \ldots, n,
$$


a system of linear equations is obtained as the matrix form $A \lambda=f$, where $a_{i j}=\varphi\left(r_{i j}\right)$ and $r_{i j}=\left\|x_{i}-x_{j}\right\|_{2}$. If the Multiquadric (MQ) radial basis function $\varphi(r)=\left(1+r^{2} \varepsilon^{2}\right)^{1 / 2}$ is used, the method is called MQ-RBF approximation. In this paper, the MQ is applied throughout the methods and examples.

In MQ-RBF approximation, $u^{*}(x)$ is presented as the following form:

$$
u^{*}(x)=\sum_{j=1}^{N} \lambda_{j}\left(1+r_{j}^{2} \varepsilon^{2}\right)^{1 / 2}
$$

where $r_{j}=\left\|x-x_{j}\right\|_{2}$ and $\varepsilon$ is the shape parameter. Sometimes, to show the dependence of $\varepsilon$ in the accuracy of $u^{*}$, the approximate function is written as $u^{*}(x ; \varepsilon)$.

Now consider a boundary value problem defined by

$$
\begin{array}{ll}
L u(x)=f(x), & \text { on } \Omega, \\
B u(x)=g(x), & \text { in } \partial \Omega,
\end{array}
$$

where $L$ is a linear differential operator and $B$ is a linear boundary operator. $\Omega$ is the domain of the problem and $\partial \Omega$ is the boundary of the domain. Suppose that $\left\{x_{i} \mid i=1, \ldots, N\right\}$ are a set of $N$ distinct centre nodes in the domain such that $\left\{x_{i} \mid i=1, \ldots, N_{1}\right\}$ are interior centre nodes and $\left\{x_{i} \mid i=\right.$ $\left.N_{1}+1, \ldots, N\right\}$ are boundary centres.

By substituting the approximation of $u$ given by RBF approximation (1), problem (4) can be written as

$$
\begin{aligned}
& \sum_{j=1}^{N} \lambda_{j} L \varphi\left(\left\|x-x_{j}\right\|\right)=f(x), \\
& \sum_{j=1}^{N} \lambda_{j} B \varphi\left(\left\|x-x_{j}\right\|\right)=g(x) .
\end{aligned}
$$

By substituting the centres $\left\{x_{i} \mid i=1, \ldots, N_{1}\right\}$ and $\left\{x_{i} \mid\right.$ $\left.i=N_{1}+1, \ldots, N\right\}$ in (5) and (6), respectively, the system of equations $H \lambda=F$ is obtained, where

$$
\begin{aligned}
& H=\left[\begin{array}{l}
L \varphi \\
B \varphi
\end{array}\right], \\
& F=\left[\begin{array}{l}
f \\
g
\end{array}\right] .
\end{aligned}
$$

And the entries are defined as follows:

$$
\begin{aligned}
& H_{i j}=\left\{\begin{array}{ll}
L \varphi\left(\left\|x_{i}-x_{j}\right\|\right), & i=1, \ldots, N_{1}, \\
B \varphi\left(\left\|x_{i}-x_{j}\right\|\right), & i=N_{1}+1, \ldots, N,
\end{array} \quad \text { for } j=1, \ldots, N,\right. \\
& F_{i}= \begin{cases}f\left(x_{i}\right), & i=1, \ldots, N_{1}, \\
g\left(x_{i}\right), & i=N_{1}+1, \ldots, N .\end{cases}
\end{aligned}
$$

By solving the system of equations $H \lambda=F$, the unknown variables $\lambda_{i}(i=1, \ldots, N)$ are determined.
2.1. Variable Shape Parameter Strategies. The shape parameter in radial basis function approximation is usually a predefined constant parameter. It is acknowledged that in many cases using variable shape parameter strategies results in more accurate approximation $[16,17,19]$. A variable shape parameter strategy uses different values of the shape parameter at different centre points:

$$
u^{*}(x)=\sum_{j=1}^{N} \lambda_{j}\left(1+r_{j}^{2} \varepsilon_{j}^{2}\right)^{1 / 2}
$$

where $\varepsilon_{j}$ is the shape parameter corresponding to the data point $x_{j}$. Applying interpolation conditions (2) to $u^{*}(x)$ results in the following system:

$$
A \lambda=f,
$$

where in $A$, the matrix of interpolation, each column has the same shape parameter, corresponding to each centre. In MQ$\mathrm{RBF}$, the interpolation matrix is as follows:

$$
A=\left[\begin{array}{ccc}
\sqrt{1+r_{11}^{2} \varepsilon_{1}^{2}} & \cdots & \sqrt{1+r_{1 n}^{2} \varepsilon_{n}^{2}} \\
\vdots & \ddots & \vdots \\
\sqrt{1+r_{n 1}^{2} \varepsilon_{1}^{2}} & \cdots & \sqrt{1+r_{n n}^{2} \varepsilon_{n}^{2}}
\end{array}\right] .
$$

Kansa and Hon [21] showed that the coefficient matrices in global radial basis function approximation tend to become progressively more ill-conditioned, but the variable shape parameter can improve the conditioning of the coefficient matrix. In fact, using variable shape parameter leads to more distinct entries in the RBF matrices which in turn lead to lower condition numbers.

Although variable shape parameter strategy is very accurate, the coefficient matrix is no longer symmetric. This is the cost that should be paid for improving the accuracy.

In variable shape parameter, $\varepsilon_{j}$ should be varied in a predetermined interval $\left[\varepsilon_{\min }, \varepsilon_{\max }\right]$. There are different approaches to distribute the values of the variable shape parameter over an interval. Kansa and Carlson presented some variable shape parameter strategies with different shape parameters at different centres [16]. Their distributions of parameters are in the forms of increasing linearly, decreasing linearly, and exponentially varying shape parameter, respectively, as follows:

$$
\begin{aligned}
& \varepsilon_{j}=\varepsilon_{\min }+\left(\frac{\varepsilon_{\max }-\varepsilon_{\min }}{N-1}\right) \times j, \\
& \varepsilon_{j}=\varepsilon_{\max }-\left(\frac{\varepsilon_{\min }-\varepsilon_{\max }}{N-1}\right) \times j, \\
& \varepsilon_{j}=\left[\varepsilon_{\min }^{2}\left(\frac{\varepsilon_{\max }^{2}}{\varepsilon_{\min }^{2}}\right)^{(j-1) /(N-1)}\right]^{1 / 2} .
\end{aligned}
$$

Their empirical results demonstrated that in comparison with the constant shape parameter the variable shape parameter can significantly reduce the RMS errors of MQ interpolations. 
They had shown that variable form of the MQ improves the accuracy of the interpolant in the interior regions where the fitted function varies relatively rapidly [16].

Sarra and Sturgill introduced a random variable shape parameter strategy in interpolations and two-dimensional linear elliptic boundary value problems as follows [19]:

$$
\varepsilon_{j}=\varepsilon_{\min }+\left(\varepsilon_{\max }-\varepsilon_{\min }\right) \times \operatorname{rand}(1, N),
$$

where rand $(1, N)$ is a Matlab function that returns $1 \times N$ matrix of pseudorandom values induced from a uniform distribution on the unit interval. They showed that the random variable shape parameter produces the most accurate results, if the centres are uniformly spaced.

Recently, some other strategies have been applied to generalized Multiquadric RBF, $\left(\left(x-x_{j}\right)^{2}+c_{j}^{2}\right)^{\beta_{j}}$. In [20] trigonometric variable shape parameter $c$ and exponent $\beta$ are presented as follows:

$$
\begin{gathered}
\beta_{j}=\beta_{\min }+\left(\beta_{\max }-\beta_{\min }\right) \times \sin (j), \quad j=1, \ldots, n, \\
c_{j}=c_{\min }+\left(c_{\max }-c_{\min }\right) \times \sin (j), \quad j=1, \ldots, n .
\end{gathered}
$$

Another strategy for variable shape parameter has been introduced by Sanyasiraju and Satyanarayana [18]. They introduced a meshless scheme with a variable shape parameter for steady convection-diffusion equation. They minimized an error function to generate a variable shape parameter for each centre node.

\section{Selection of an Interval in Variable Shape Parameter}

In addition to selecting a suitable strategy, there is another important problem in variable shape parameter approach: distribution of the values in an appropriate interval. In [20], by using the classical variable MQ as $\left(c_{j}^{2}+r^{2}\right)^{\beta}$, an interval such as $\left[c_{\min }, c_{\max }\right]$ is introduced, where $c_{\min }=1 / \sqrt{N}$ and $c_{\max }=3 / \sqrt{N}$ (the shape parameter of classical form of the MQ is shown by $c$ ). $N$ is the total number of nodes in onedimensional approximations and is the node number of a row or a column in two-dimensional approximations. Sarra and Sturgill emphasize the importance of the length of the interval, $k=\varepsilon_{\max }-\varepsilon_{\min }$, they take $k=1$, and they study the errors by trial and errors, while $\varepsilon_{\max }$ and $\varepsilon_{\min }$ vary in a meaningful range [19]. Kansa and Carlson [16] consider a set of shape parameters which minimizes an error function over some evaluation points. To find these values, the following objective function is minimized:

$$
z=\sum_{m=1}^{M}\left\|f_{\text {exact }}\left(\bar{x}_{m}\right)-f_{\mathrm{MQ}}\left(\bar{x}_{m}\right)\right\|^{2}
$$

where

$$
f_{\mathrm{MQ}}\left(\bar{x}_{m}\right)=\sum_{j=1}^{N} \lambda_{j}\left(\left\|\bar{x}_{m}-x_{j}\right\|+c_{j}^{2}\right)^{1 / 2}
$$

and $\bar{x}_{m}(m=1, \ldots, M)$ are evaluation points to compute the RMS error. They used two approaches to find the optimal set for the shape parameters. In the first approach, a nonlinear system of normal equations is obtained by least square approach:

$$
\frac{\partial z}{\partial c_{j}}=0, \quad j=1, \ldots, N
$$

In the second one, the sum of squares over the evaluation points is minimized. For more details refer to [16].

In $[19,20]$, it is seen that the proposed values for $\varepsilon_{\max }$ and $\varepsilon_{\min }$ are empirical. In Kansa's approach a set of optimal shape parameters has been obtained, but the shortage of this approach is that the exact function $f_{\text {exact }}$ is needed. Kansa stated that the purpose of his report was some empirical observations to developments in the theory of the variable shape parameters. Therefore his approach is not applicable in practice. In this paper, we apply a new algorithm to find a reliable interval for the shape parameters.

3.1. Proposed Algorithm. In this section the algorithm is introduced.

Suppose that $u^{*}(x ; \varepsilon)=\sum_{j=1}^{n} \lambda_{j} \varphi\left(r_{j}\right)$ approximates the function $u(x)$, over a domain $D$. In Section 2 , it was noted that the accuracy of $u^{*}(x ; \varepsilon)$ depends on $\varepsilon$. For all valid values of $\varepsilon$, keeping $x^{*} \in D$ fixed, the corresponding values of $u^{*}\left(x^{*} ; \varepsilon\right)$ should be convergent to $u\left(x^{*}\right)$. Therefore

$$
u^{*}(x ; \varepsilon) \longrightarrow u(x), \quad \forall x \in D .
$$

If the curve $C$ is the plot of $u^{*}\left(x^{*} ; \varepsilon\right)$ as a function of the shape parameter $\varepsilon$ versus the shape parameter, then $C$ will be horizontal over an interval, say $R_{\varepsilon}$. This interval is a suitable range for the valid values of the shape parameter. According to this idea, the proposed algorithm to find an appropriate interval for the shape parameter can be summarized as follows.

\section{Algorithm}

(1) Let $\varepsilon=\left[\varepsilon_{1}, \varepsilon_{2}, \ldots, \varepsilon_{N}\right]^{t}$.

(2) For $k=1$ to $N$.

(3) Plot the curve of $u^{*}$ versus $\varepsilon$.

Do evaluate the value of $u^{*}\left(x^{*} ; \varepsilon_{k}\right)$.

(4) Select the subinterval $R_{\varepsilon}$, over which the curve is almost parallel to $\varepsilon$ axis.

The interval $R_{\varepsilon}$ or a subset of this interval can be applied in the variable shape parameter strategies as $\left[\varepsilon_{\min }, \varepsilon_{\max }\right]$. The approximate function is recomputed by using the proposed interval.

\section{Numerical Examples}

In this section to demonstrate the reliability and the efficiency of the algorithm in various types of variable shape parameter strategies, three examples, for interpolation in one- and two-dimensional spaces, are presented. Also two boundary value problems in two-dimensional spaces are investigated. Through all the examples we use the uniform centre nodes. 


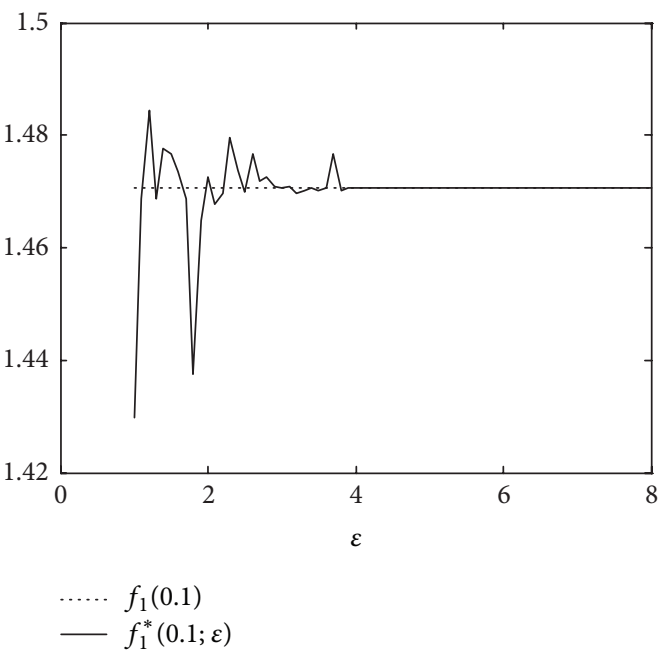

(a)

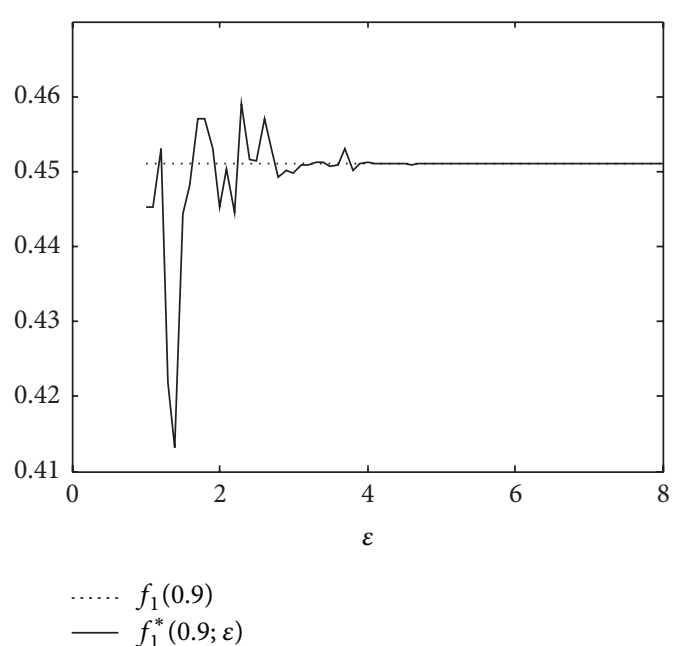

(c)

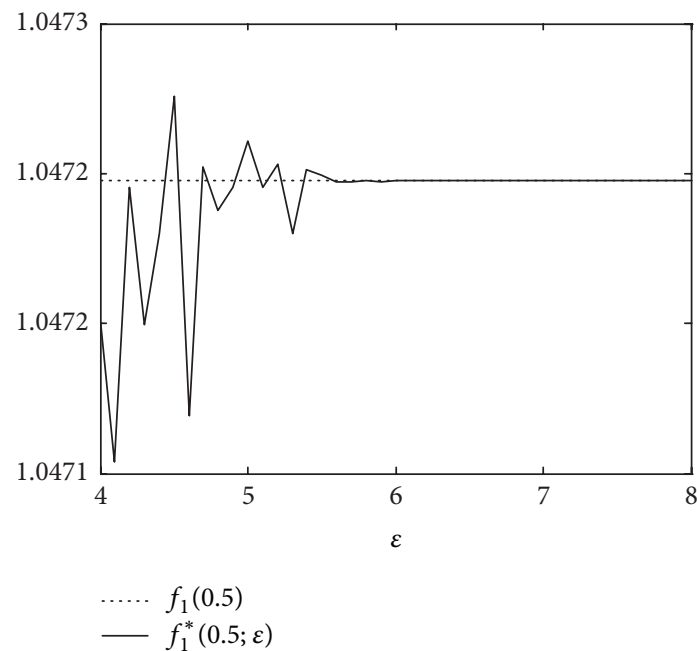

(e)

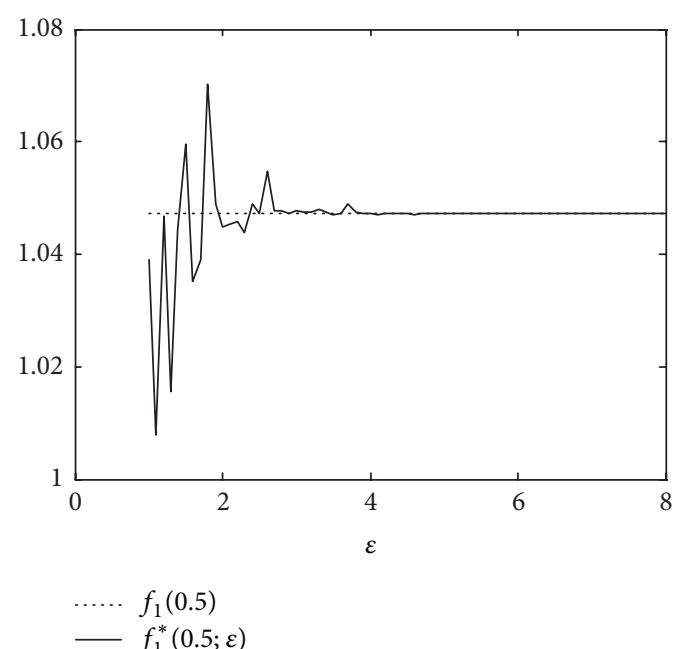

(b)

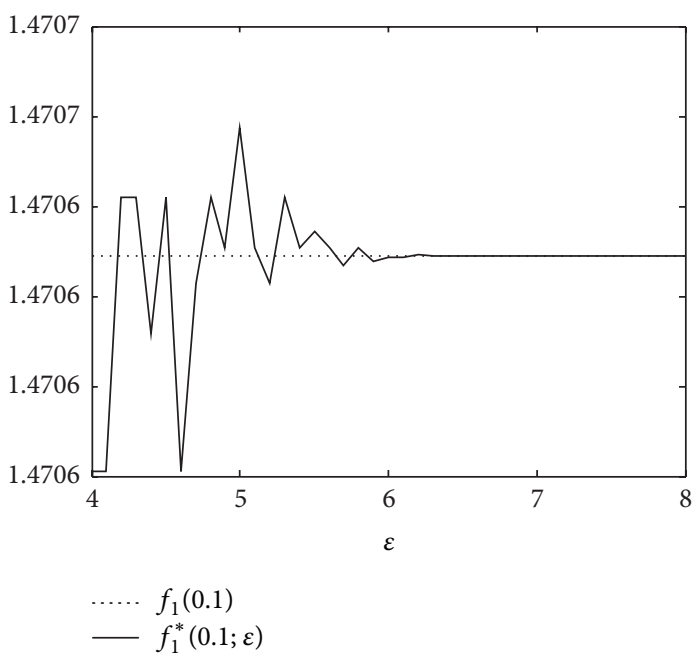

(d)

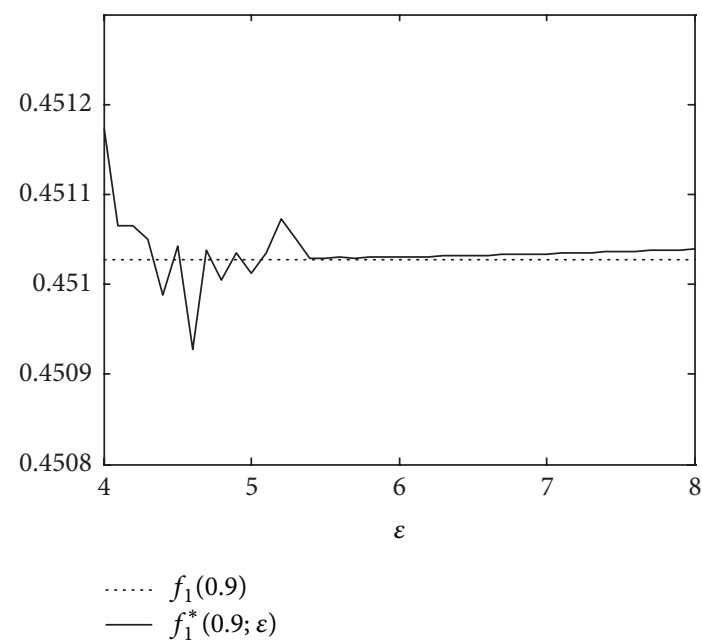

(f)

Figure 1: The plots of $f_{1}^{*}\left(x^{*} ; \varepsilon\right)$ for $x^{*}=0.1, x^{*}=0.5$, and $x^{*}=0.9(\mathrm{a}, \mathrm{b}$, and c) versus the shape parameters $\varepsilon \in[1,8]$ and $\varepsilon \in[4,8](\mathrm{d}, \mathrm{e}$, and $\mathrm{f}$ ). 


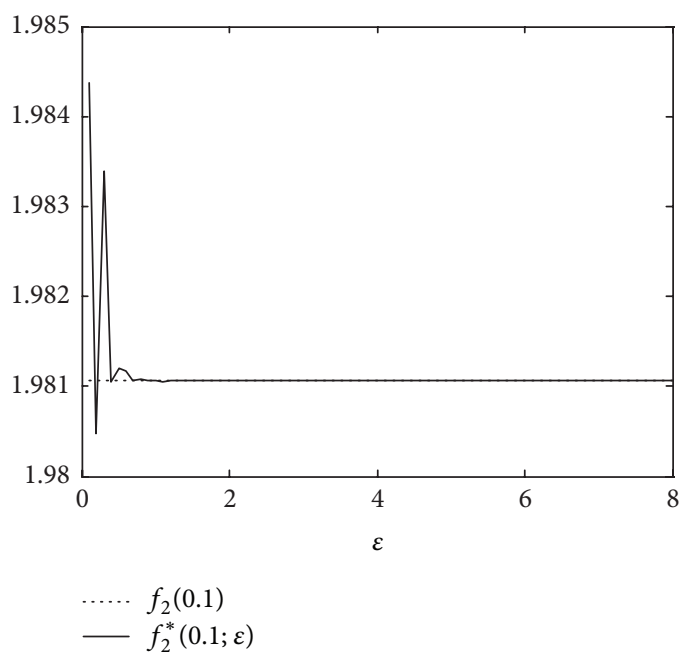

(a)

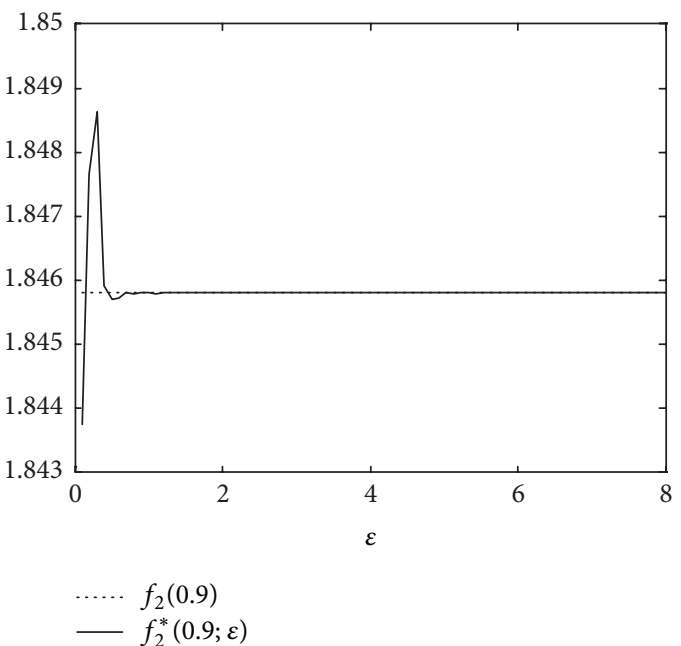

(c)

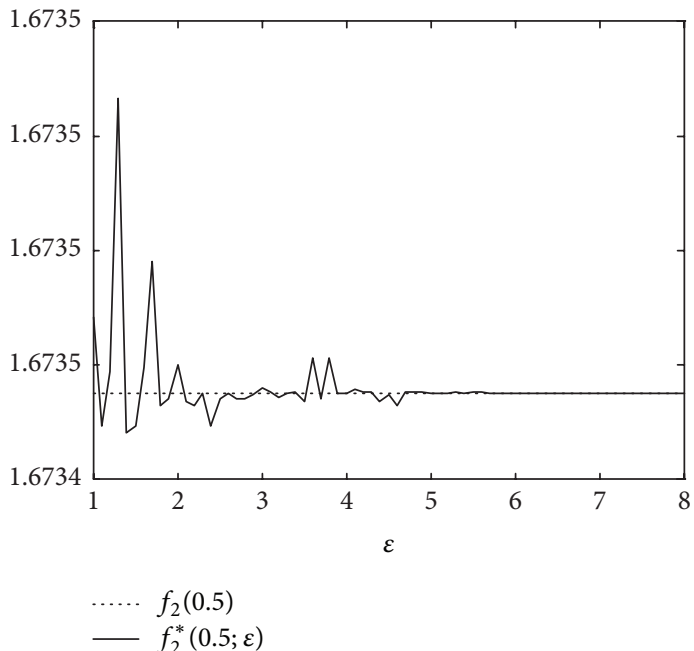

(e)

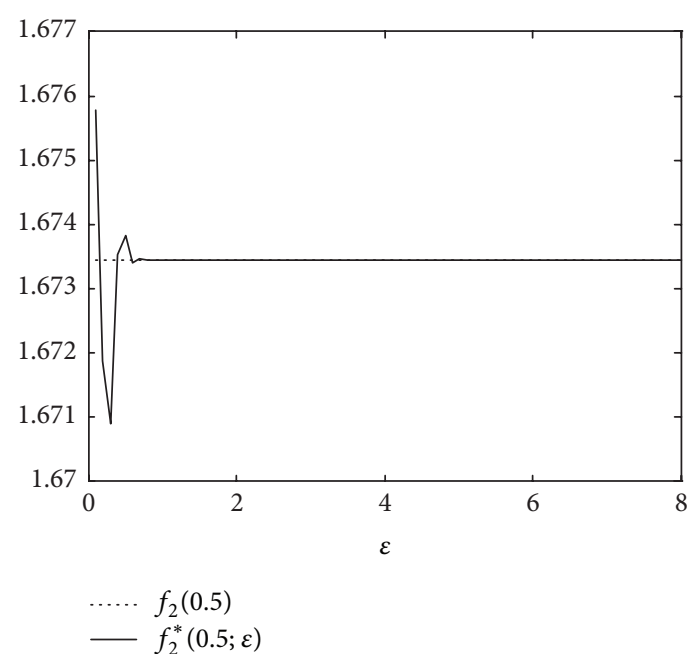

(b)

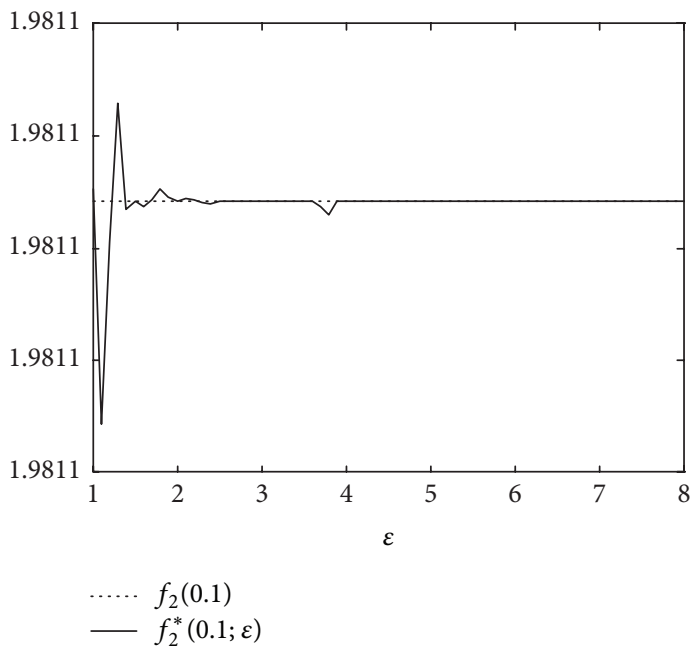

(d)

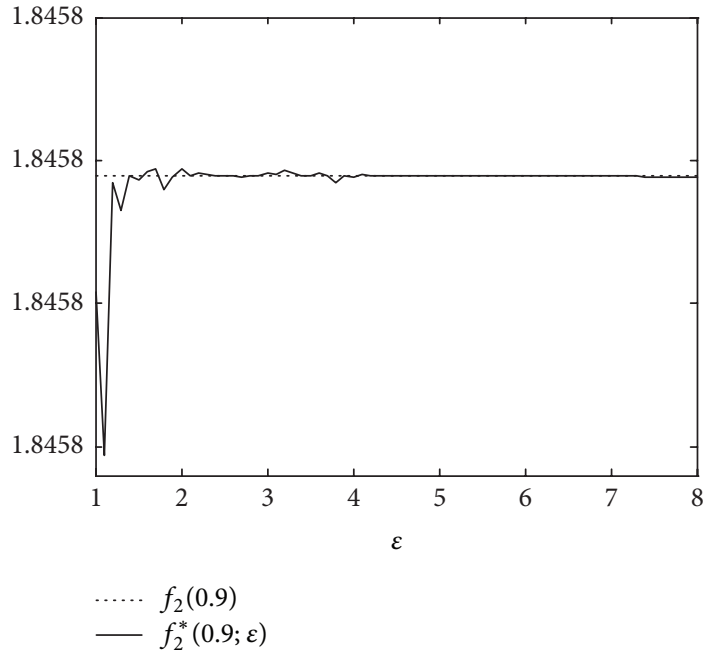

(f)

Figure 2: The plots of $f_{2}^{*}\left(x^{*} ; \varepsilon\right)$ for $x^{*}=0.1, x^{*}=0.5$, and $x^{*}=0.9(\mathrm{a}, \mathrm{b}$, and $\mathrm{c})$ versus the shape parameters $\varepsilon \in[0.1,8]$ and $\varepsilon \in[1,8](\mathrm{d}, \mathrm{e}$, and $\mathrm{f}$ ). 


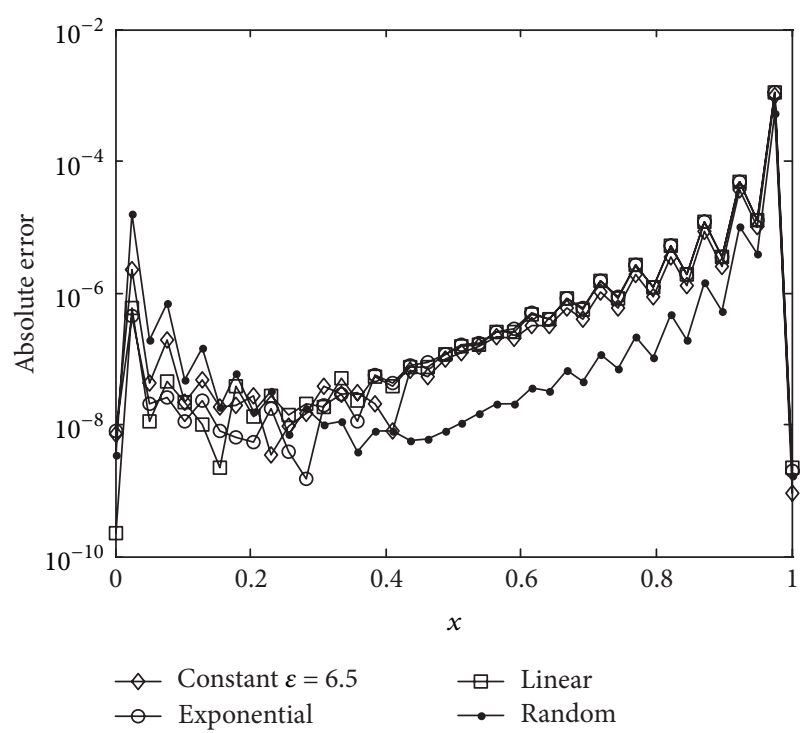

(a)

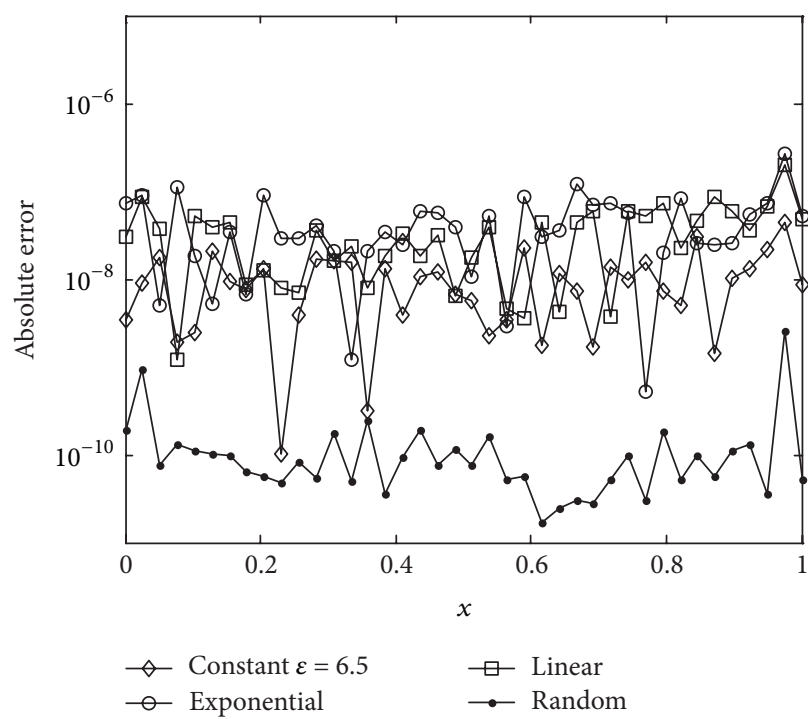

(b)

FIGURE 3: The error functions for approximation of $f_{1}(x)(\mathrm{a})$ and $f_{2}(x)$ (b) by constant, exponential, linear, and random shape parameters in the proposed intervals.

To validate the numerical accuracy, two error functions such as Max error and RMS error are calculated:

$$
\begin{aligned}
\text { Max-Error } & =\max _{1 \leq i \leq M}\left(\left|u^{*}\left(x_{i}\right)-f\left(x_{i}\right)\right|\right), \\
\text { RMS-Error } & =\sqrt{\frac{1}{M} \sum_{i=1}^{M}\left(u^{*}\left(x_{i}\right)-f\left(x_{i}\right)\right)^{2},}
\end{aligned}
$$

where $x_{i}(i=1, \ldots, M)$ are some evaluation points.

In random variable shape parameter strategy, due to the nature of random selection, the results may be different. In all of the numerical results, to reduce this impact, the average error of ten time successive runs is provided, when the random variable shape parameter strategy is used.

4.1. One- and Two-Dimensional Interpolation. The test functions in one-dimensional interpolation are

$$
\begin{aligned}
& f_{1}(x)=\arccos (x), \\
& f_{2}(x)=e^{x^{3}}+\cos (2 x),
\end{aligned}
$$

$$
x \in[0,1] .
$$

First, the algorithm is applied to find an interval for each test function and then the approximate function is recomputed by using the variable shape parameter strategy in the proposed interval. To compare the results, the obtained accuracy is compared with the accuracy of approximate functions computed by using variable shape parameter in different intervals.

Figures $1(\mathrm{a}), 1(\mathrm{~b})$, and $1(\mathrm{c})$ show the curve of $f_{1}^{*}\left(x^{*} ; \varepsilon\right)$ versus shape parameter $\varepsilon$ in the interval $[1,8]$ for three different $x^{*} \in D=[0,1]$, using 60 centre nodes. The curve is almost parallel to $\varepsilon$ axis, over the interval $[4,8]$. The interval can be selected as $\left[\varepsilon_{\min }, \varepsilon_{\max }\right] \subseteq[4,8]$, in variable shape parameter. By rerunning the "plot command" on subinterval $[4,8]$, a more accurate interval is obtained by Figures $1(\mathrm{~d})$, $1(\mathrm{e})$, and $1(\mathrm{f})$. By considering $\varepsilon_{\min }=6$ and $\varepsilon_{\max }=7$, the error functions in interpolation of $f_{1}$ by constant, exponential, linear, and random shape parameter strategies are plotted in Figure 3(a). 40 evaluation points and 60 centre points are applied. In this figure, the impact of different distribution strategies is shown. To illustrate the influence of the interval $\left[\varepsilon_{\min }, \varepsilon_{\max }\right]$, which is the aim of this study, the results of applying the proposed interval for two strategies (random and exponential) are shown in Figure 4.

The plots in Figure 4 show that the proposed interval obtained by the algorithm is reliable and accurate for variable shape parameter strategies. By selecting the shape parameters in the proposed range the accuracy significantly improved.

By visual inspection, in Figures 2(a), 2(b), and 2(c), $f_{2}^{*}\left(x^{*} ; \varepsilon\right)$ as a function of the shape parameter $\varepsilon$ has the same value in the interval $[1,8]$. By rerunning the plots in $[1,8]$, Figures $2(\mathrm{~d}), 2(\mathrm{e})$, and $2(\mathrm{f})$ demonstrate that the suitable interval can be selected as $[6,7]$. Using the interval $\left[\varepsilon_{\min }, \varepsilon_{\max }\right]=[6,7]$ in variable shape parameter, the error functions obtained by constant, exponential, linear, and random shape parameter strategies are shown in Figure 3(b). To demonstrate the reliability of this interval, the error functions obtained by exponential and random variable shape parameter in the intervals $[0.5,1.5],[2,3],[4,5]$, and $[6,7]$ are plotted in Figure 4 . The errors demonstrate that using the proposed interval $[6,7]$, obtained by the proposed algorithm, results in better accuracy.

It is noted that, for various test points $x^{*}$, the optimum range may be a little different. The experimental results show that the proposed interval may not be the optimal one to approximate in the whole domain, but it is a valid interval. Therefore, in next examples, the results of only one test point $x^{*} \in D$ are plotted. 


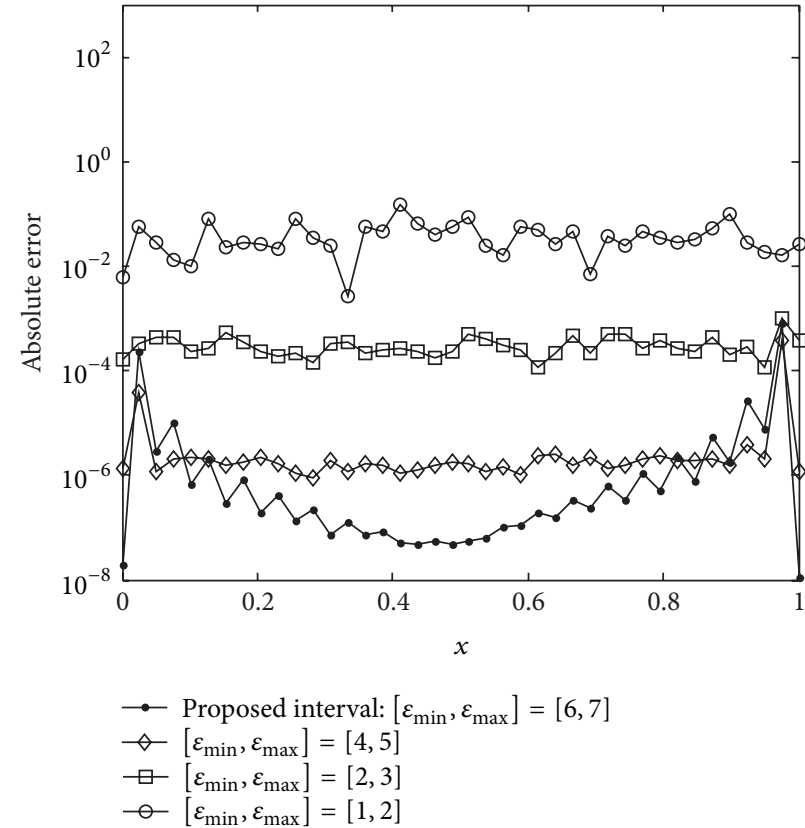

(a)

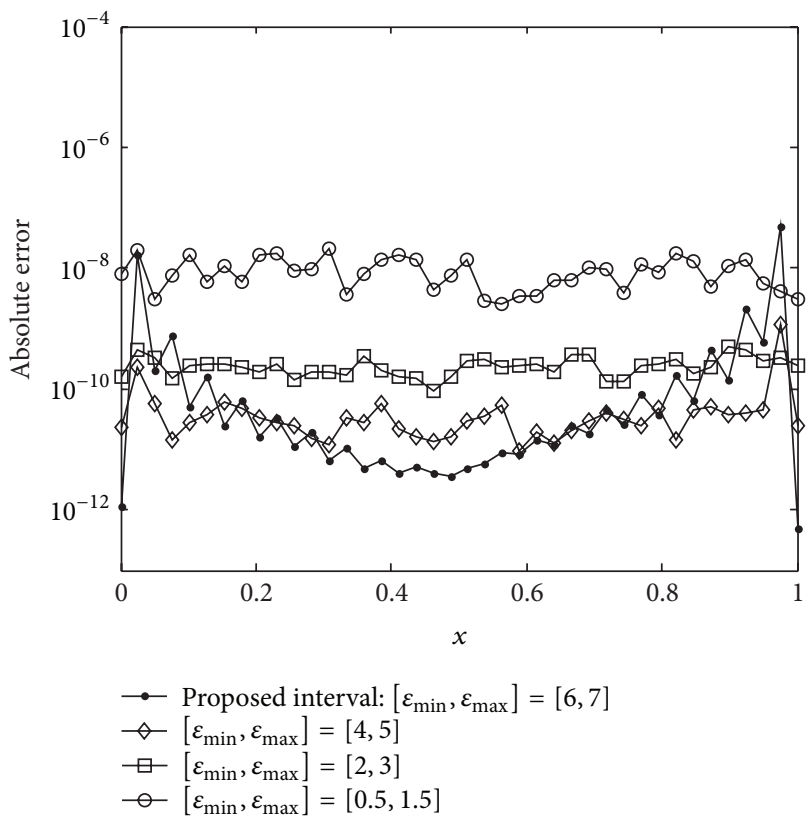

(c)

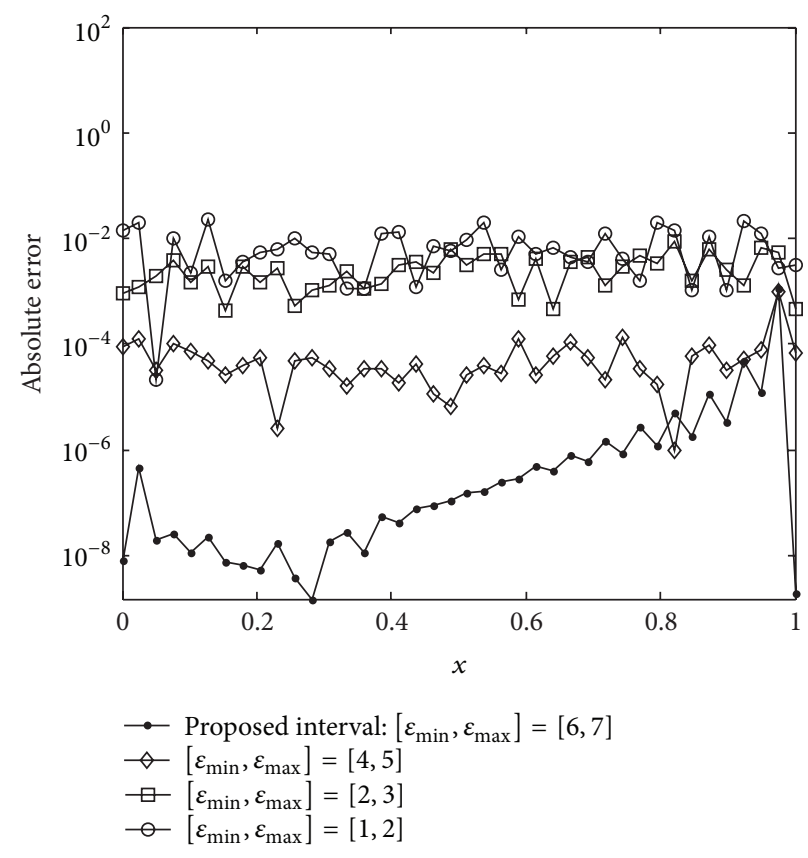

(b)

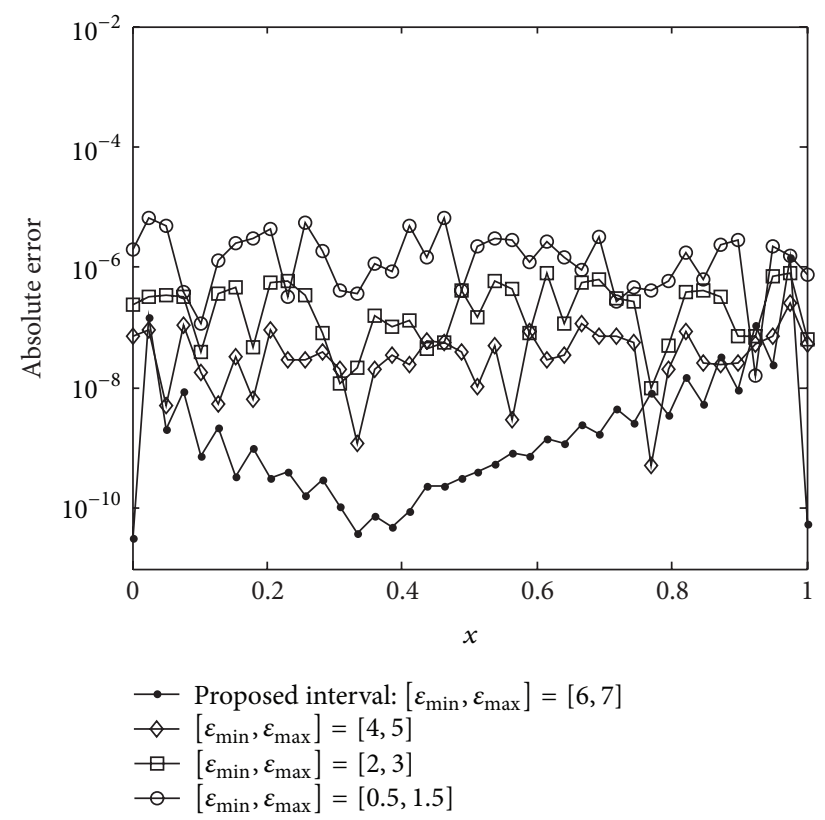

(d)

FIGURE 4: The error functions by random $(\mathrm{a}, \mathrm{c})$ and exponential $(\mathrm{b}, \mathrm{d})$ variable shape parameters in different intervals for approximating $f_{1}(x)$ $(\mathrm{a}, \mathrm{b})$ and $f_{2}(x)(\mathrm{c}, \mathrm{d})$. tion

In two-dimensional interpolation consider the test func-

$$
f_{3}(x, y)=e^{x+2 y}
$$

in the rectangle $D=[-0.5,0.5] \times[-0.5,0.5]$. Using 144 centre points, the algorithm is applied for an arbitrary point $\left(x^{*}, y^{*}\right)=(0.2778,0.0556) \in D$. Figure $5(a)$ shows the curve obtained in step (3) of the algorithm. The plot demonstrates that the appropriate interval can be chosen as $\left[\varepsilon_{\min }, \varepsilon_{\max }\right]=$ $[0.5,1.5]$. The error function of approximate function by using linear variable shape parameter in this interval is shown in Figure 6. Also some numerical results are summarized in Table 1.

4.2. Boundary Value Problems. To illustrate the accuracy and the efficiency of the algorithm, two linear boundary 
TABLE 1: The comparison of the RMS error and Max error for different intervals.

\begin{tabular}{|c|c|c|c|c|c|c|}
\hline & \multirow{2}{*}{\multicolumn{2}{|c|}{$\left[\varepsilon_{\min }, \varepsilon_{\max }\right]$}} & \multicolumn{2}{|c|}{ Exponential } & \multicolumn{2}{|c|}{ Random } \\
\hline & & & RMS error & Max error & RMS error & Max error \\
\hline \multirow{4}{*}{$f_{3}$} & Proposed interval & {$[0.5,1.5]$} & $4.79 e-6$ & $2.32 e-5$ & $7.15 e-7$ & $1.72 e-6$ \\
\hline & & {$[1,2]$} & $1.00 e-4$ & $4.61 e-4$ & $2.89 e-5$ & $6.84 e-5$ \\
\hline & Some other intervals & {$[3,4]$} & $4.10 e-4$ & $2.29 e-3$ & $1.56 e-3$ & $5.44 e-3$ \\
\hline & & {$[4,5]$} & $6.81 e-4$ & $3.74 e-3$ & $6.35 e-4$ & $3.32 e-3$ \\
\hline \multirow{4}{*}{ Problem (12) } & Proposed interval & {$[0.4,1]$} & $2.69 e-5$ & $7.82 e-5$ & $5.13 e-5$ & $9.22 e-5$ \\
\hline & & {$[1,2]$} & $1.50 e-3$ & $2.84 e-3$ & $9.28 e-4$ & $2.02 e-3$ \\
\hline & Some other intervals & {$[2,3]$} & $2.01 e-3$ & $3.57 e-3$ & $4.69 e-3$ & $9.07 e-3$ \\
\hline & & {$[4,5]$} & $4.93 e-3$ & $7.58 e-3$ & $4.73 e-3$ & $7.02 e-3$ \\
\hline \multirow{4}{*}{ Problem (13) } & Proposed interval & {$[0.2,1]$} & $2.40 e-9$ & $6.86 e-9$ & $2.25 e-12$ & $5.09 e-12$ \\
\hline & & {$[1,2]$} & $1.17 e-4$ & $2.04 e-4$ & $1.04 e-6$ & $1.76 e-6$ \\
\hline & Some other intervals & {$[2,3]$} & $1.67 e-4$ & $3.03 e-4$ & $7.23 e-4$ & $2.56 e-3$ \\
\hline & & {$[3,4]$} & $4.18 e-4$ & $6.70 e-4$ & $1.17 e-3$ & $2.25 e-3$ \\
\hline
\end{tabular}

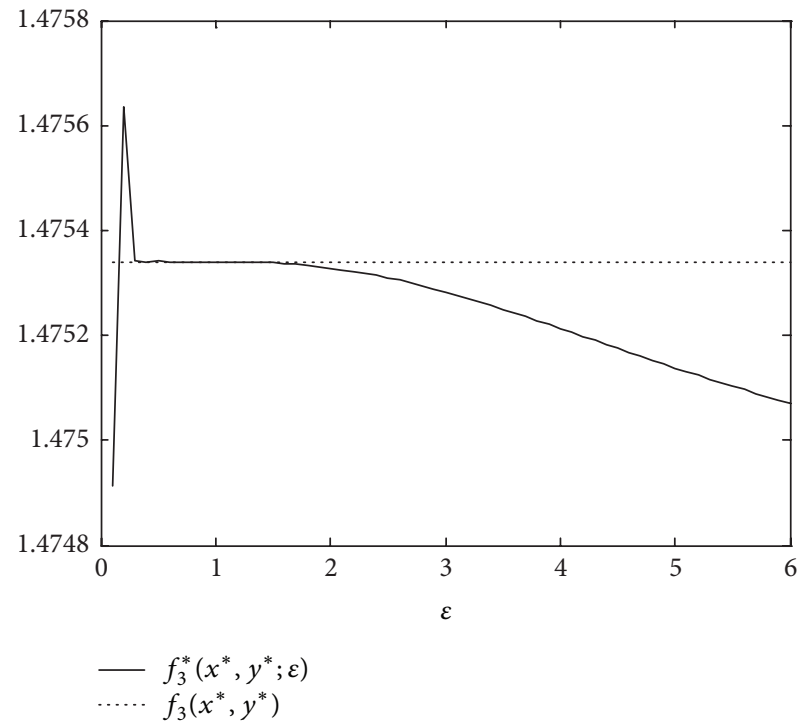

(a)

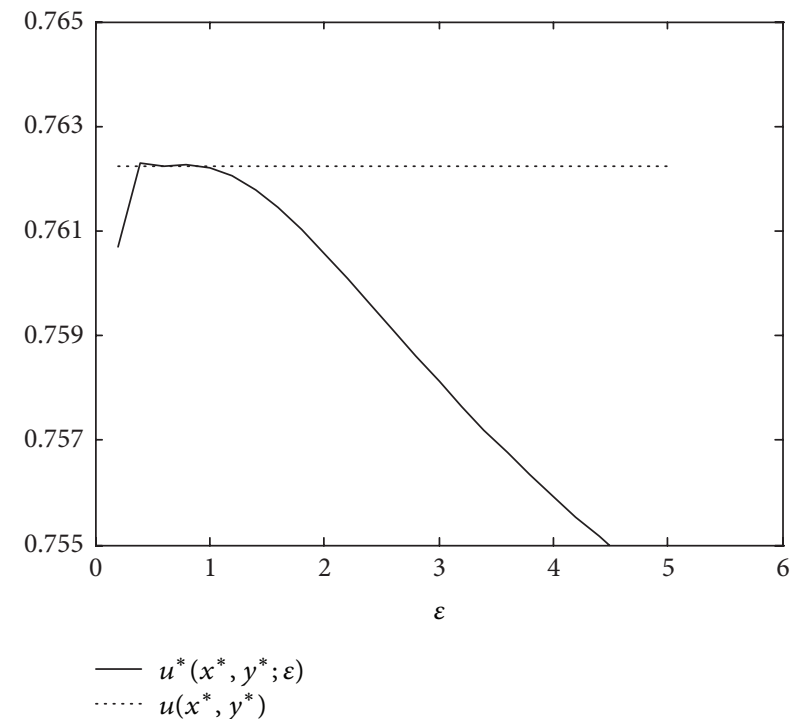

(b)

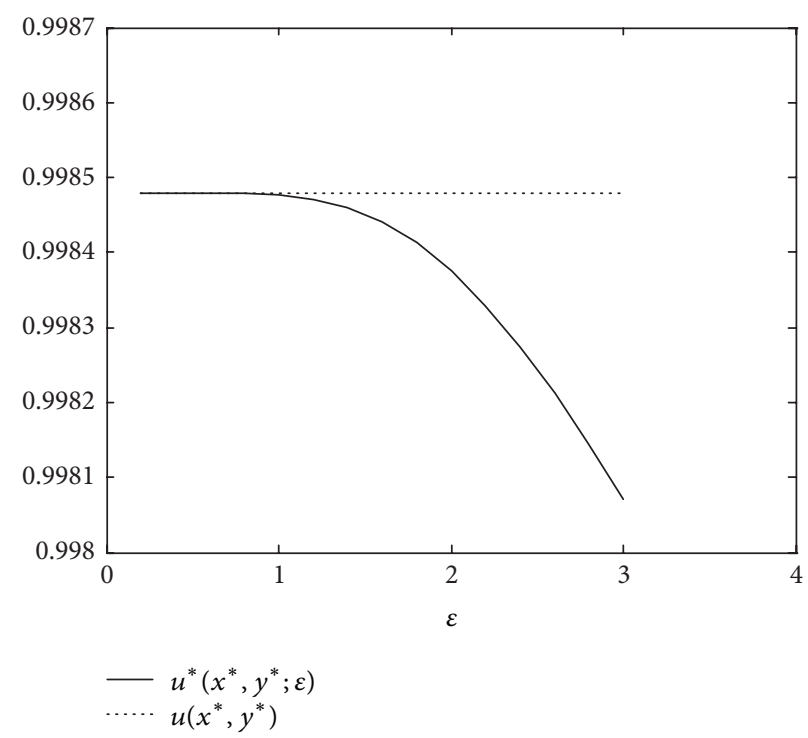

(c)

Figure 5: Plot of $f_{3}^{*}\left(x^{*}, y^{*} ; \varepsilon\right)$ (a) and the solution of problems (12) and (13), $u^{*}\left(x^{*}, y^{*} ; \varepsilon\right)$ (resp., (b) and (c)), versus the shape parameter $\varepsilon$. 


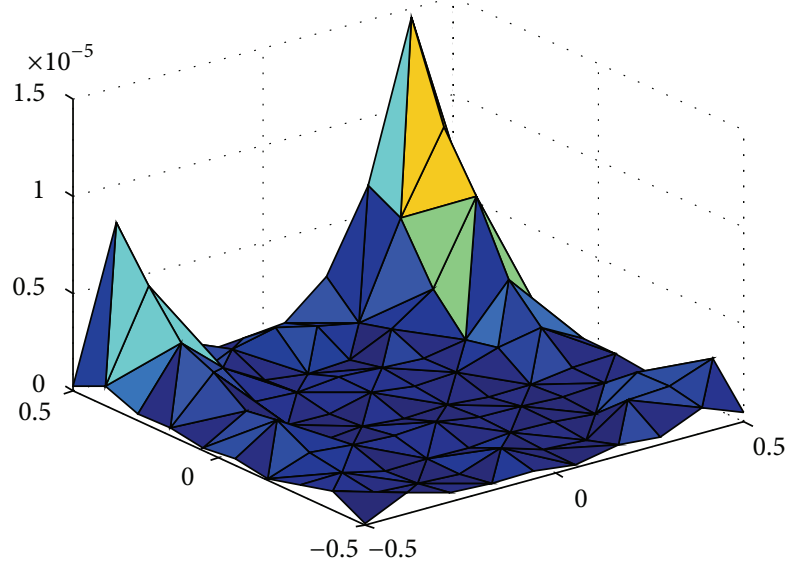

(a)

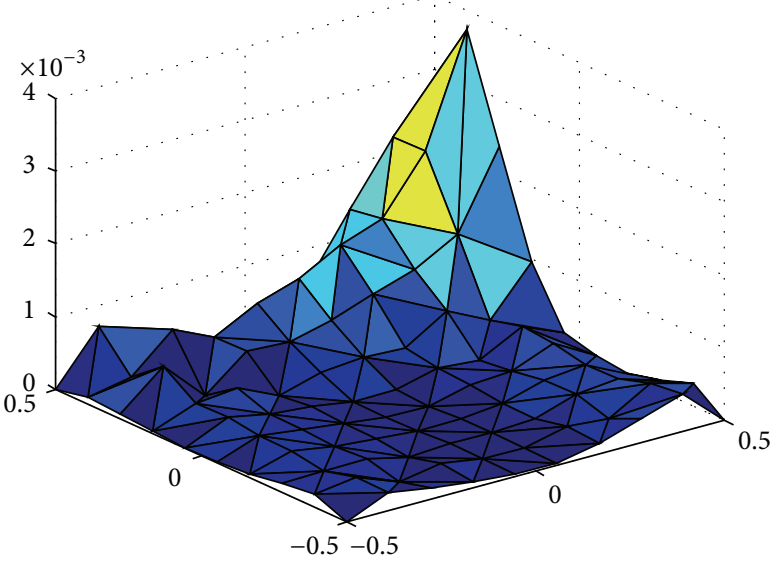

(b)

FIGURE 6: The error function of the approximation of $f_{3}(x, y)$ by linear variable shape parameter in the intervals $[0.5,1.5](a)$ and $[4,5](b)$.

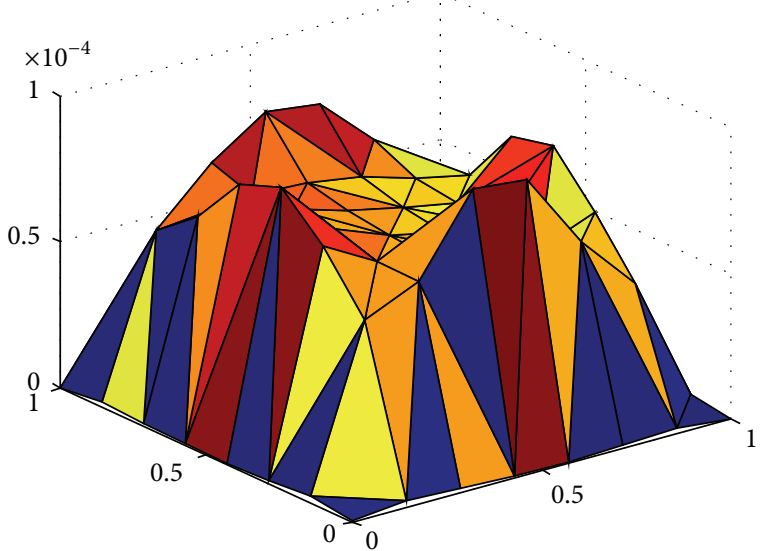

(a)

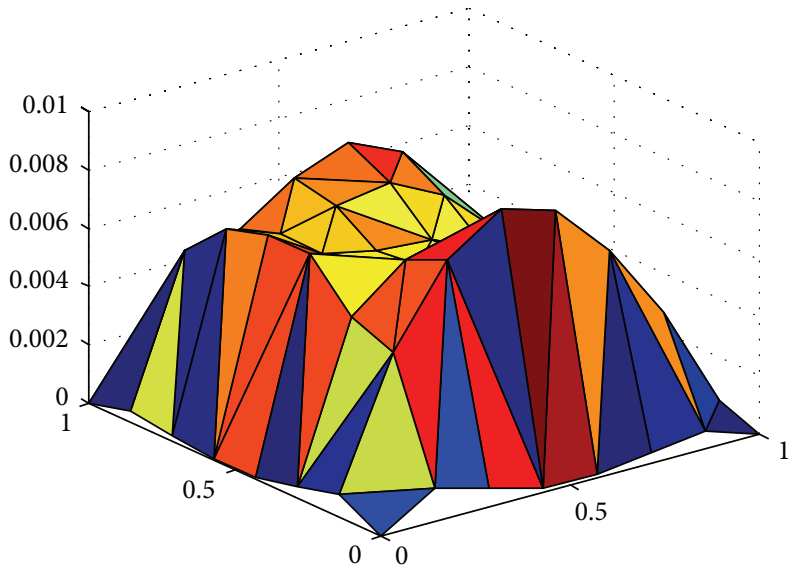

(b)

FIGURE 7: The error function of the approximation of problem (24) by random variable shape parameters in intervals $[0.4,1](\mathrm{a})$ and $[2,3]$ (b).

value problems are considered. First consider the following BVP:

$$
\begin{aligned}
u_{x x}+u_{y y} & =-2 \pi^{2} \sin (\pi x) \cos (\pi y), \quad(x, y) \in \Omega, \\
u(x, y) & =0, \quad(x, y) \in \Gamma=\partial \Omega,
\end{aligned}
$$

where $\Omega=[0,1] \times[0,1]$ and $\Gamma$ is the boundary of the domain. The exact solution is $u(x, y)=\sin (\pi x) \cos (\pi y)$. Using 100 centre points, the algorithm is applied to approximate the solution of problem (24). The plot of $u\left(x^{*}, y^{*}\right)$, for a predetermined $\left(x^{*}, y^{*}\right)=(0.7143,0.5714) \in \Omega$, versus shape parameter $\varepsilon$ is shown in Figure 5(b). The curve determines the appropriate interval $\left[\varepsilon_{\min }, \varepsilon_{\max }\right]=[0.4,1]$. The comparison of the error functions by applying this interval to various shape parameter strategies with those obtained by some other intervals is shown in Figure 7 and Table 1. Numerical results demonstrated that one can achieve good numerical results by choosing the interval $[0.4,1]$ determined by the algorithm.
The algorithm is also performed for the following problem:

$$
\begin{aligned}
& u_{x x}+u_{y y}=f(x, y), \\
& \quad(x, y) \in \Omega=[-0.5,0.5] \times[-0.5,0.5], \\
& u(x, y)=g(x, y), \quad(x, y) \in \Gamma=\partial \Omega,
\end{aligned}
$$

where $f$ and $g$ are specified such that the exact solution is

$$
u(x, y)=\frac{65}{65+(x-0.2)^{2}+(y+0.1)^{2}}
$$

Using 100 centre points and an arbitrary point $\left(x^{*}, y^{*}\right)=$ $(0.2143,0.2143) \in \Omega$, Figure 5(c) illustrates that the interval $[0.2,1]$ can be selected for applying in the variable shape parameter strategies. The absolute error of the approximate solution by applying this interval, which has been shown in Figure 8 , indicates that the proposed interval significantly 


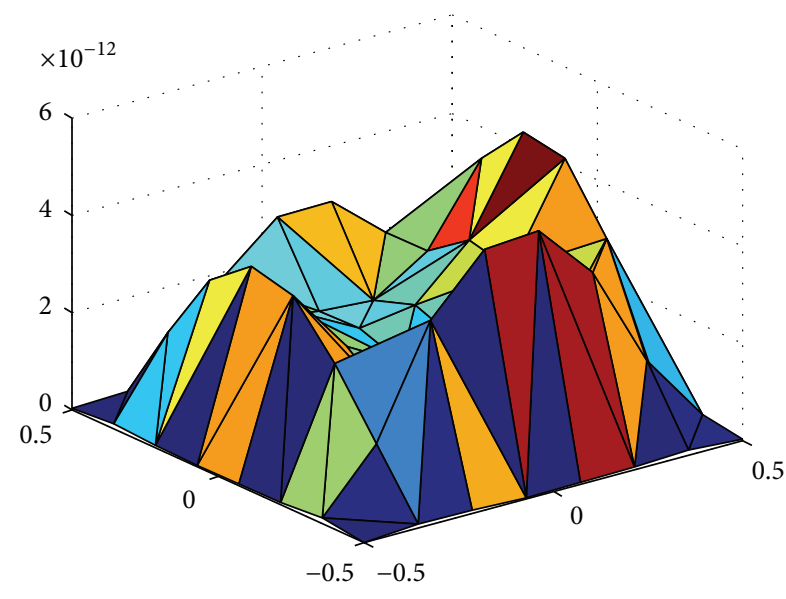

(a)

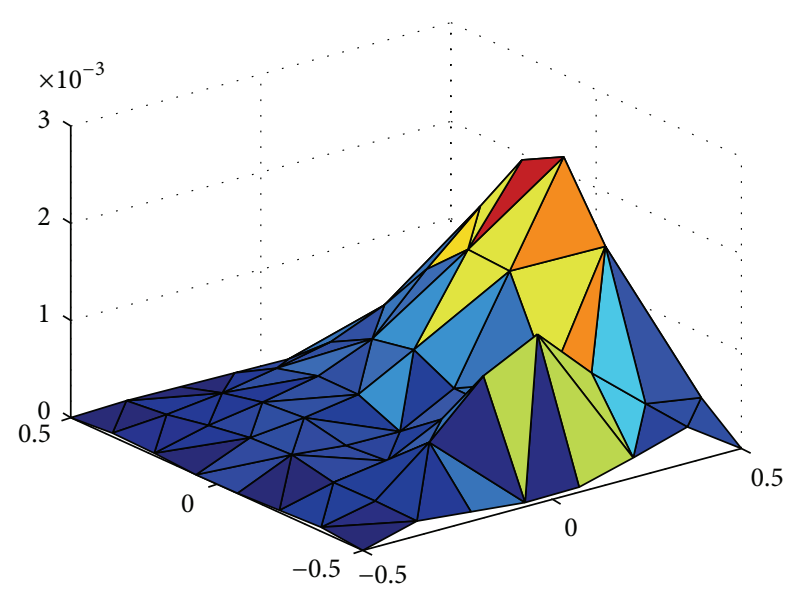

(b)

FIGURE 8: The error function of the approximation of problem (25) by random variable shape parameter in intervals $[0.2,1](\mathrm{a})$ and $[2,3](\mathrm{b})$.

increases the accuracy of the results. The results of Table 1 illustrate the impact of applying a suitable interval in different variable shape parameter strategies.

\section{Conclusion}

In this paper, a new algorithm is suggested to determine an interval for variable shape parameter strategies. By distributing the values of the shape parameters in the proposed interval, it was shown that the new numerical results will be more accurate. To show the efficiency of the proposed interval for different strategies, some famous variable shape parameter strategies were investigated. The results demonstrate that in one- and two-dimensional interpolation problems and in two-dimensional boundary value problems the algorithm works well.

\section{Competing Interests}

The authors declare that there is no conflict of interests regarding the publication of the paper.

\section{References}

[1] G. E. Fasshauer, Meshfree Approximation Methods with MAT$L A B$, vol. 6, World Scientific, River Edge, NJ, USA, 2007.

[2] A. J. M. Ferreira, L. M. S. Castro, and S. Bertoluzza, "A high order collocation method for the static and vibration analysis of composite plates using a first-order theory," Composite Structures, vol. 89, no. 3, pp. 424-432, 2009.

[3] Q. Shen, "A meshless method of lines for the numerical solution of $\mathrm{KdV}$ equation using radial basis functions," Engineering Analysis with Boundary Elements, vol. 33, no. 10, pp. 1171-1180, 2009.

[4] J. Lin, W. Chen, and K. Y. Sze, "A new radial basis function for Helmholtz problems," Engineering Analysis with Boundary Elements, vol. 36, no. 12, pp. 1923-1930, 2012.

[5] Z.-J. Fu, W. Chen, and L. Ling, "Method of approximate particular solutions for constant- and variable-order fractional diffusion models," Engineering Analysis with Boundary Elements, vol. 57, pp. 37-46, 2015.

[6] R. E. Carlson and T. A. Foley, "The parameter R2 in multiquadric interpolation," Computers and Mathematics with Applications, vol. 21, no. 9, pp. 29-42, 1991.

[7] S. Rippa, "An algorithm for selecting a good value for the parameter c in radial basis function interpolation," Advances in Computational Mathematics, vol. 11, no. 2-3, pp. 193-210, 1999.

[8] V. Bayona, M. Moscoso, and M. Kindelan, "Optimal constant shape parameter for multiquadric based RBF-FD method," Journal of Computational Physics, vol. 230, no. 19, pp. 7384-7399, 2011.

[9] A. H. D. Cheng, "Multiquadric and its shape parameter-a numerical investigation of error estimate, condition number, and round-off error by arbitrary precision computation," Engineering Analysis with Boundary Elements, vol. 36, no. 2, pp. 220239, 2012.

[10] G. E. Fasshauer and J. G. Zhang, “On choosing 'optimal' shape parameters for RBF approximation," Numerical Algorithms, vol. 45, no. 1-4, pp. 345-368, 2007.

[11] B. Fornberg and G. Wright, "Stable computation of multiquadric interpolants for all values of the shape parameter," Computers \& Mathematics with Applications, vol. 48, no. 5-6, pp. 853-867, 2004.

[12] C.-S. Huang, C.-F. Lee, and A. H.-D. Cheng, "Error estimate, optimal shape factor, and high precision computation of multiquadric collocation method," Engineering Analysis with Boundary Elements, vol. 31, no. 7, pp. 614-623, 2007.

[13] C. M. Roque and A. J. Ferreira, "Numerical experiments on optimal shape parameters for radial basis functions," Numerical Methods for Partial Differential Equations, vol. 26, no. 3, pp. 675689, 2010.

[14] C. H. Tsai, J. Kolibal, and M. Li, "The golden section search algorithm for finding a good shape parameter for meshless collocation methods," Engineering Analysis with Boundary Elements, vol. 34, no. 8, pp. 738-746, 2010.

[15] M. Uddin, "On the selection of a good value of shape parameter in solving time-dependent partial differential equations using RBF approximation method," Applied Mathematical Modelling, vol. 38, no. 1, pp. 135-144, 2014. 
[16] E. J. Kansa and R. E. Carlson, "Improved accuracy of multiquadric interpolation using variable shape parameters," Computers \& Mathematics with Applications, vol. 24, no. 12, pp. 99120, 1992.

[17] B. Fornberg and J. Zuev, “The Runge phenomenon and spatially variable shape parameters in RBF interpolation," Computers \& Mathematics with Applications, vol. 54, no. 3, pp. 379-398, 2007.

[18] Y. Sanyasiraju and C. Satyanarayana, "Local Hermite-RBF based grid-free scheme with a variable (optimal) shape parameter for steady convection-diffusion equations," International Journal of Numerical Analysis and Modeling, Series B, vol. 4, pp. 382-393, 2013.

[19] S. A. Sarra and D. Sturgill, "A random variable shape parameter strategy for radial basis function approximation methods," Engineering Analysis with Boundary Elements, vol. 33, no. 11, pp. 1239-1245, 2009.

[20] S. Xiang, K.-M. Wang, Y.-T. Ai, Y.-D. Sha, and H. Shi, “Trigonometric variable shape parameter and exponent strategy for generalized multiquadric radial basis function approximation," Applied Mathematical Modelling, vol. 36, no. 5, pp. 1931-1938, 2012.

[21] E. J. Kansa and Y. C. Hon, "Circumventing the Ill-conditioning problem with multiquadric radial basis functions: applications to elliptic partial differential equations," Computers \& Mathematics with Applications, vol. 39, no. 7-8, pp. 123-137, 2000. 


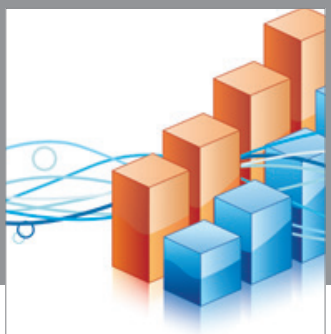

Advances in

Operations Research

vatem alat4

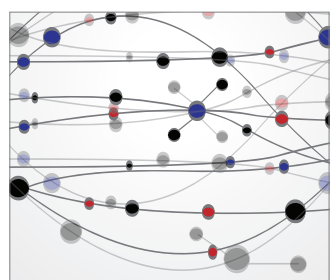

\section{The Scientific} World Journal
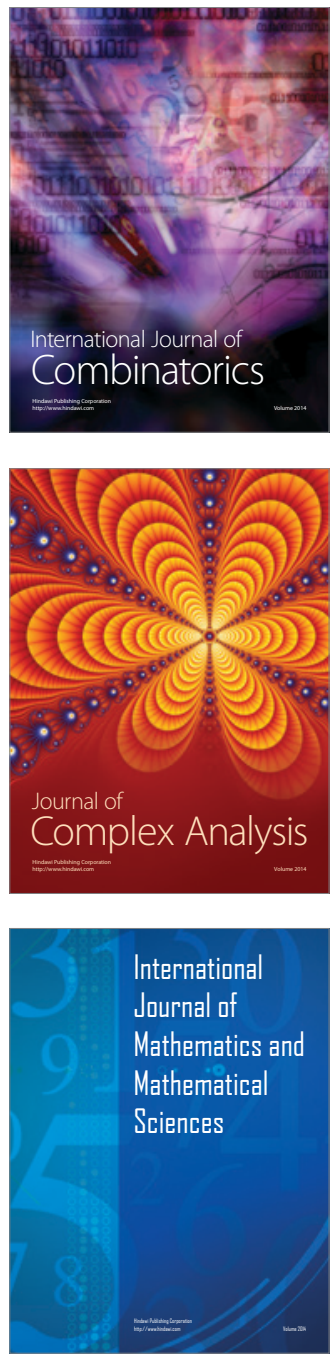
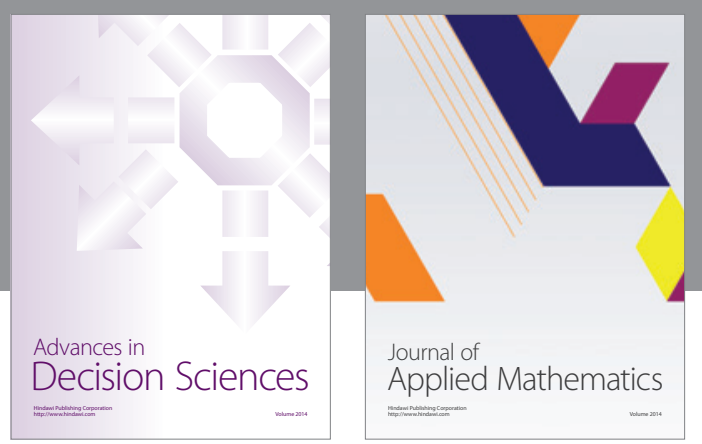

Algebra

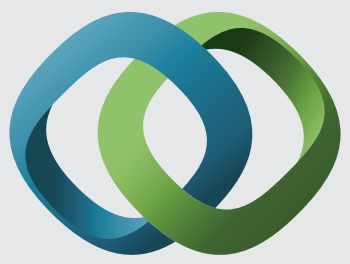

\section{Hindawi}

Submit your manuscripts at

http://www.hindawi.com
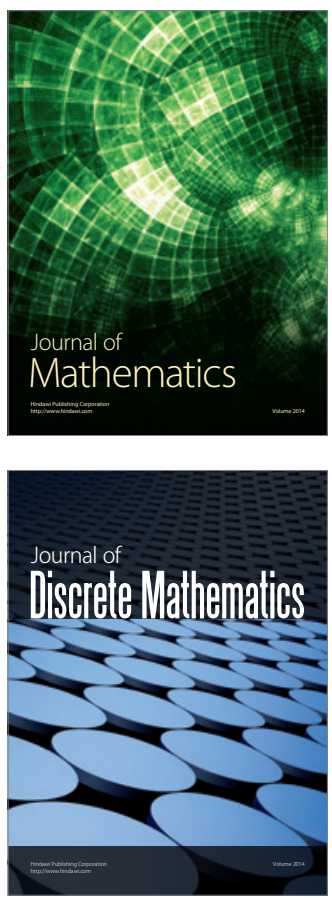

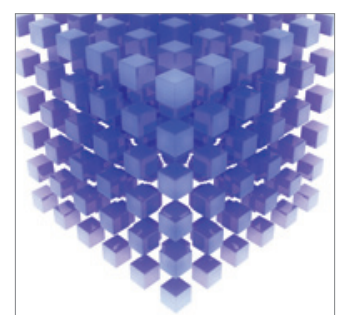

Mathematical Problems in Engineering
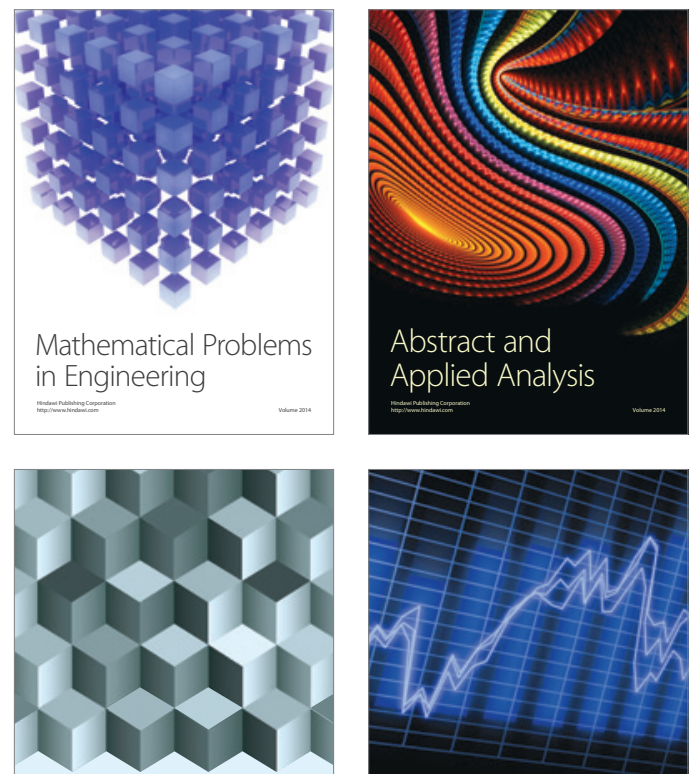

Journal of

Function Spaces

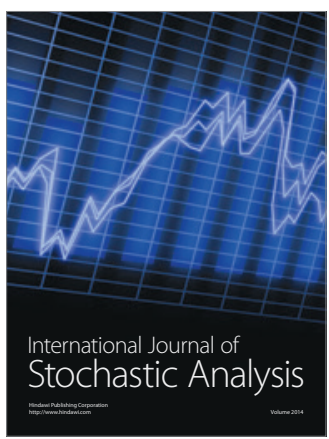

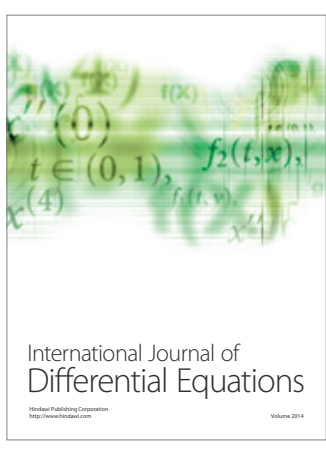
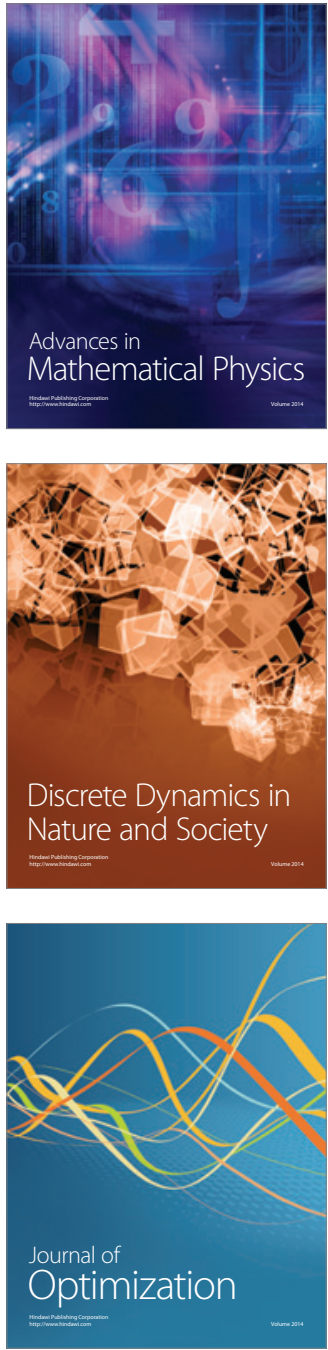